\title{
氧化还原沉淀法制备 $\mathrm{MnO}_{2} / \mathrm{MWCNTs}$ 催化剂 及其低温 SCR 活性
}

\author{
陈健, 郑玉婴, 张延兵, 邹海强, 卢秀恋 \\ (福州大学 材料科学与工程学院, 福州 350108)
}

\begin{abstract}
摘 要: 先用十二烷基硫酸钠(SDS)对多壁碳纳米管(MWCNTs)进行表面改性, 然后采用氧化还原沉淀法制得一系 列的 $\mathrm{MnO}_{2} / \mathrm{MWCNTs}$ 催化剂。考察了催化剂在 $80 \sim 180^{\circ} \mathrm{C}$ 的选择性催化还原(SCR)反应活性, 并通过 BET、XRD、 FESEM、TEM、XPS 和 $\mathrm{H}_{2}$-TPR 等表征手段对催化剂的结构及性能进行分析。结果显示, $\mathrm{MnO}_{2} / \mathrm{MWCNTs}$ 催化剂在 空速 $210 \mathrm{~L} /\left(\mathrm{g}_{\mathrm{cat}} \cdot \mathrm{h}\right)$ 和温度 $140 \sim 180^{\circ} \mathrm{C}$ 条件下, 脱硝效率达到 $85 \%$ 100\%, 这明显优于等体积浸渍法制备的催化剂的 低温 SCR 催化活性，且 $10 \% \mathrm{MnO}_{2} / \mathrm{MWCNTs}$ 催化剂的活性最优。分析结果表明, $\mathrm{MnO}_{2} / \mathrm{MWCNTs}$ 催化剂中 $\mathrm{MnO}_{2}$ 以纳米片状均匀分散在多壁碳纳米管载体表面; 弱结晶性的结构和高价锰, 较高的表面吸附含氧量及较强的低温 区氧化还原能力是 $10 \% \mathrm{MnO}_{2} / \mathrm{MWCNTs}$ 催化剂具有优异低温 SCR 活性的原因。另外, 和 $\mathrm{MnO}_{x} / \mathrm{MWCNTs}$ 催化剂 相比, $10 \% \mathrm{MnO}_{2} / \mathrm{MWCNTs}$ 催化剂表现出良好的抗水和抗硫性能。
\end{abstract}

关＼cjkstart键＼cjkstart词：十二烷基硫酸钠；选择性催化还原；氮氧化物；多壁碳纳米管；镇氧化物

中图分类号: TB34 文献标识码: A

\section{Preparation of $\mathrm{MnO}_{2} / \mathrm{MWCNTs}$ Catalysts by a Redox Method and Their Activity in Low-temperature SCR}

\author{
CHEN Jian, ZHENG Yu-Ying, ZHANG Yan-Bing, ZOU Hai-Qiang, LU Xiu-Lian \\ (College of Materials Science and Engineering, Fuzhou University, Fuzhou 350108, China)
}

\begin{abstract}
Pristine multiwalled carbon nanotubes (MWCNTs) were modified by sodium dodecyl sulfate (SDS). Afterwards, a series of $\mathrm{MnO}_{2} / \mathrm{MWCNTs}$ were prepared via a redox method and used in selective catalytic reduction (SCR) of $\mathrm{NO}_{x}$ at $80-180^{\circ} \mathrm{C}$. Structural properties and catalytic performance were investigated by specific surface area measurements (BET), X-ray diffraction (XRD), filed emission scanning electron microscope (FESEM), transmission electron microscope (TEM), X-ray photoelectron spectroscope (XPS), and $\mathrm{H}_{2}$ temperature-programmed reduction $\left(\mathrm{H}_{2}\right.$-TPR). The characterization results indicate that the nanoflaky $\mathrm{MnO}_{2}$ highly disperse on MWCNTs. The results show that the low-temperature SCR activities of $\mathrm{MnO}_{2} / \mathrm{MWCNTs}$ catalysts are $85 \%-100 \%$ at $140-180^{\circ} \mathrm{C}$ at a weight hourly space velocity of $210 \mathrm{~L} /\left(\mathrm{g}_{\mathrm{cat}} \mathrm{h}\right)$, higher than that of catalyst fabricated via the wet impregnation method. The $10 \% \mathrm{MnO}_{2}$ /MWCNTs catalyst displays first-rate SCR activity, which is attributed to its low crystallinity, high concentrations of $\mathrm{Mn}^{4+}$ and oxygen absorbtion onto the catalyst surface, as well as excellent reducibility. Additionally, compared to the $\mathrm{MnO}_{x} / \mathrm{MWCNTs}$ catalyst, the $10 \% \mathrm{MnO}_{2}$ /MWCNTs catalyst exhibits higher resistance to $\mathrm{H}_{2} \mathrm{O}$ and $\mathrm{SO}_{2}$.
\end{abstract}

Key words: sodium dodecyl sulfate; selective catalytic reduction; nitrogen oxide; multi-walled carbon nanotube; manganese oxide 
氮氧化物 $\left(\mathrm{NO}_{x}\right)$ 是主要的大气污染物, 能引起 雾皬、光化学烟雾、臭氧层破坏及一系列人体健康 问题 ${ }^{[1]}$ 。当前应用最广泛、技术最成熟的治理工业 源 $\mathrm{NO}_{x}$ 的技术是以氨气 $\left(\mathrm{NH}_{3}\right)$ 为还原剂的选择性催 化还原法(Selective catalytic reduction, $\mathrm{SCR})^{[2-3]}$ 。 $\mathrm{SCR}$ 技术去除 $\mathrm{NO}_{x}$ 的关键材料是催化剂, 目前商 用钒钛催化剂 $\mathrm{V}_{2} \mathrm{O}_{5} / \mathrm{TiO}_{2}$ 在中温段 $\left(300 \sim 400^{\circ} \mathrm{C}\right)$ 催 化性能优越 ${ }^{[4]}$, 在该温度段脱硝设备被置于脱硫 除尘装置之前, 该催化剂在高灰量和高 $\mathrm{SO}_{2}$ 环境 下容易失活 ${ }^{[2,5]}$, 因此理想的脱硝工艺是将 SCR 脱硝设备置于脱硫设备和除尘装置之后, 但是此 时的烟气温度会降低至 $200^{\circ} \mathrm{C}$ 以下 ${ }^{[6]}$ 。为了避免中 温催化剂需要再加热烟气浪费能耗, 开发高效的 且能适应低温活性窗口 $\left(80 \sim 200^{\circ} \mathrm{C}\right)$ 的催化剂具有 非常重要的意义。

锰基催化剂在 $\mathrm{NH}_{3}-\mathrm{SCR}$ 反应中有较强的低温 催化性, 因而低温 $\mathrm{NH}_{3}$-SCR 催化剂的研究主要集中 在氧化锰基催化剂。Kapteijn 等 ${ }^{[7]}$ 测试了一系列纯锰 氧化物 $\left(\mathrm{MnO}_{x}\right)$ 的 $\mathrm{NH}_{3}-\mathrm{SCR}$ 活性, 发现其催化活性 和 $\mathrm{N}_{2}$ 选择性由氧化物状态和结晶度决定, 其中 $\mathrm{MnO}_{2}$ 的单位面积活性最高, $\mathrm{Mn}_{2} \mathrm{O}_{3}$ 的 $\mathrm{N}_{2}$ 选择性最 高。但是, 制备的无载体 $\mathrm{MnO}_{x}$ 催化剂存在自身结 构不稳定、比表面积小及低温活性不理想等缺点。 因此, 研究各种负载型 $\mathrm{MnO}_{x}$ 催化剂, 使其具有优良 的低温反应活性和催化效率是解决这些问题的有力 措施, 如 $\mathrm{MnO}_{x} / \mathrm{CeO}_{2}{ }^{[8]} 、 \mathrm{MnO}_{x} / \mathrm{TiO}_{2}{ }^{[9]} 、 \mathrm{MnO}_{x} / \mathrm{Al}_{2} \mathrm{O}_{3}{ }^{[10]}$ 和 $\mathrm{MnO}_{x} / \mathrm{ACF}^{[11]}$ 等催化剂。而碳纳米管因具有孔隙 发达、比表面积大、机械强度高及纳米直径等优点,
很适合作为载体负载纳米级金属或金属氧化物，在 催化剂载体方面具有广阔的应用前景 ${ }^{[12]}$ 。虽然已有相 关研究报道了碳纳米管负载镇氧化物 $\left(\mathrm{MnO}_{x} / \mathrm{CNTs}\right)$ 催 化剂, 但是它们的低温活性都集中在 $200 \sim 300^{\circ} \mathrm{C}$ 的 温度范围内, 且低温 SCR 活性均不理想。所以, 研 究和开发新型低温高效 $\mathrm{MnO}_{x} / \mathrm{CNTs}$ 催化剂具有重 要的理论和实用价值。

本工作采用新颖简单易行的氧化还原沉淀法, 制备出 $\mathrm{MnO}_{2} / \mathrm{MWCNTs}$ 系列催化剂, 研究了其低温 SCR 催化活性及抗 $\mathrm{H}_{2} \mathrm{O}$ 、抗 $\mathrm{SO}_{2}$ 性能, 并将其与传 统等体积浸渍法制备的 $\mathrm{MnO}_{x} / \mathrm{MWCNTs}$ 催化剂进 行比较, 旨在为开发高效廉价的低温 SCR 实用型催 化剂提供新的思路和方法。

\section{1 实验方法}

\section{1 催化剂的制备}

图 1 为制备 $\mathrm{MnO}_{2} / \mathrm{MWCNTs}$ 催化剂的流程图。首 先, 将 $0.3 \mathrm{~g} \mathrm{MWCNTs}$ (直径为 $60 \sim 100 \mathrm{~nm}$, 深圳纳米港 有限公司)加入到 $50 \mathrm{~mL}$ 去离子水中, 磁力搅拌一段时 间后加入 $0.0237 \mathrm{~g}$ 的 SDS; 随后将混合均匀的悬浊液 置于 $100 \mathrm{~W}$ 超声波清洗器中超声分散 $1 \mathrm{~h}$, 使得 SDS 半胶束所形成的外壳能够充分吸附在 MWCNTs 表 面, 形成荷电层 ${ }^{[13]}$; 再将一定量的浓 $\mathrm{HNO}_{3}$ (国药集 团化学试剂有限公司, AR)加入到上述悬浮液中, 并 混合摚拌 $12 \mathrm{~h}$ ，让分散在 MWCNTs 表后将体系升温 至 $70^{\circ} \mathrm{C}$ 并缓慢滴加 $20 \mathrm{~mL}$ 的 $\mathrm{KMnO}_{4}$ (国药集团化学 试剂有限公司, $\mathrm{AR}$ ) 溶液, 使 $\mathrm{KMnO}_{4}$ 在酸性溶液
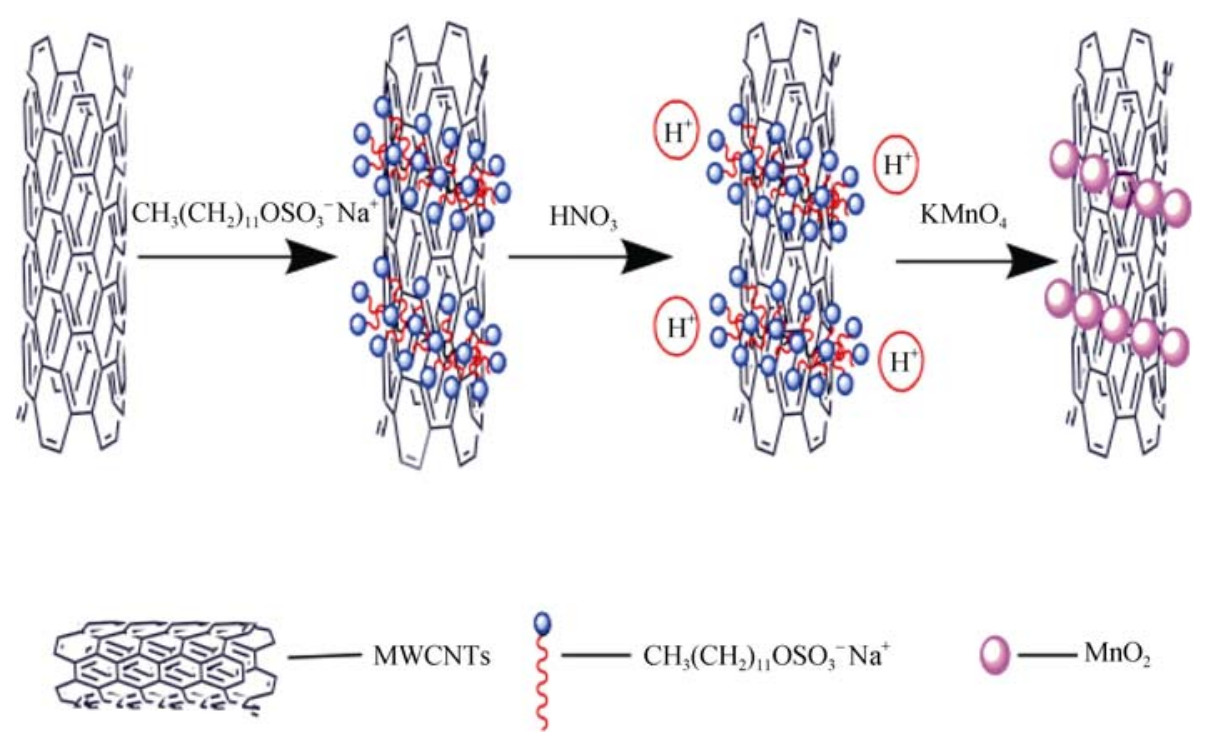

图 $1 \mathrm{MnO}_{2} / \mathrm{MWCNTs}$ 催化剂的制备示意图

Fig. 1 Schematic illustration for preparing $\mathrm{MnO}_{2} / \mathrm{MWCNTs}$ catalysts 
中与碳纳米管表面的碳反应, 消耗 $\mathrm{H}^{+}$离子的同时在 MWCNTs 表面原位沉积 $\mathrm{MnO}_{2}$ 。其中浓 $\mathrm{HNO}_{3}$ 与 $\mathrm{KMnO}_{4}$ 的摩尔比为 1: 1 , 反应时间为 $1 \mathrm{~h}$ 。 $\mathrm{KMnO}_{4}$ 和 MWCNTs 在酸性体系中的反应, 可由如下化学 方程式所示 ${ }^{[14-15]}$ :

$$
4 \mathrm{MnO}_{4}{ }^{-}+3 \mathrm{C}+4 \mathrm{H}^{+} \rightarrow 4 \mathrm{MnO}_{2}+3 \mathrm{CO}_{2}+2 \mathrm{H}_{2} \mathrm{O}
$$

最后, 将制得的 $\mathrm{MnO}_{2} / \mathrm{MWCNTs}$ 催化剂过滤, 并用无水乙醇和去离子水反复洗涤数次。得到产物 在 $105^{\circ} \mathrm{C}$ 的真空干燥箱中干燥 $12 \mathrm{~h}$ 。所得催化剂用 $Y$ $\mathrm{MnO}_{2} / \mathrm{MWCNTs}$ 表示, $Y$ 代表 $\mathrm{Mn} / \mathrm{C}$ 的摩尔比率。为 了对比, 根据参考文献[16], 采用传统等体积浸渍法, 在管式炉 $300^{\circ} \mathrm{C}$ 有氧环境下炷烧制备 $\mathrm{MnO}_{x} / \mathrm{MWCNTs}$ 催化剂 $(\mathrm{Mn} / \mathrm{C}$ 的摩尔比率为 $10 \%)$ 。

\section{2 催化剂 SCR 活性评价}

在实验室自制的不锈钢固定反应器中进行催化 剂的活性评价, 实验温度通过程序升温系统控制。 模拟烟道气体总流量为 $700 \mathrm{~mL} / \mathrm{min}$, 其中 $\mathrm{NO}$ 为 $400 \times 10^{-6}, \mathrm{NH}_{3}$ 为 $400 \times 10^{-6}, \mathrm{O}_{2}$ 占 $5 \%, \mathrm{SO}_{2}$ 为 $100 \times 10^{-6}$ (选用), $\mathrm{H}_{2} \mathrm{O}$ 为 $5 \%$ (选用), 其余为平衡气体 $\mathrm{N}_{2}$, 催化剂 用量为 $200 \mathrm{mg}$, 质量空速 (WHSV)为 $210 \mathrm{~L} /\left(\mathrm{g}_{\mathrm{cat}} \cdot \mathrm{h}\right)$ 。气 体流量由质量流量计控制, 气体在进入管式电阻炉 之前先通过气体混合器混合均匀, 再经过预热器预 热后进入反应器, 运行温度在 $80^{\circ} \mathrm{C} \sim 180^{\circ} \mathrm{C}$ 。用 KM950 烟气分析仪测定管式电阻炉中进出口气体 的成分及含量。

催化剂的脱硝活性用 $\mathrm{NO}_{x}$ 转化率来评价, 并由 下式计算得到:

$$
\delta\left(\mathrm{NO}_{x}\right)=\frac{\phi\left(\mathrm{NO}_{x}\right)_{\text {in }}-\phi\left(\mathrm{NO}_{x}\right)_{\text {out }}}{\phi\left(\mathrm{NO}_{x}\right)_{\text {in }}} \times 100 \%
$$

式中, $\delta\left(\mathrm{NO}_{x}\right)$ 为 $\mathrm{NO}_{x}$ 转化率, $\phi\left(\mathrm{NO}_{x}\right)_{\text {in }}$ 和 $\phi\left(\mathrm{NO}_{x}\right)_{\text {out }}$ 分 别为反应器进出口处 $\mathrm{NO}_{x}$ 的体积分数。

\section{3 催化剂的表征}

采用美国 Micromeritics 公司的 ASAP 2020 M 全自动比表面积及微孔孔隙分析仪测定催化剂的比

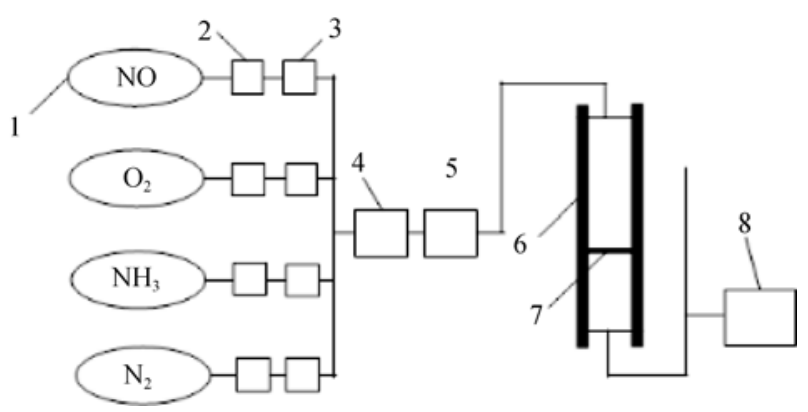

图 2 实验装置示意图

Fig. 2 Scheme of experiment apparatus

1. Air source; 2. Reducing valve; 3. Mass flowmeter; 4. Gas mixer;

5. Preheater; 6. Heater; 7. Sample; 8. Flue gas analyzer
表面积和孔容孔径, 样品测试前经 $350^{\circ} \mathrm{C}$ 真空处理 $4 \mathrm{~h}$, 以 $\mathrm{N}_{2}$ 为吸附质, 在 $-196^{\circ} \mathrm{C}$ 下进行测定。采用日本 Rigaku 公司的 Dmax/3C 型 X 射线多晶衍射仪 $(\mathrm{Cu} \mathrm{K \alpha}$ 射线, $\lambda=0.15406 \mathrm{~nm}$ )测定样品物相, 操作电压为 $40 \mathrm{kV}$, 操作电流为 $20 \mathrm{~mA}$, 扫描速度为 $8 \% \mathrm{~min}$, 扫描角度 $10^{\circ} \sim 80^{\circ}$ 。在美国 FEI 公司 Nova NanoSEM 230 型 场发射扫描电子显微镜下对催化剂表面形态进行 观察。在美国 FEI 公司 Tecnai G2F20 型透射电子 显微镜下观测催化剂样品微观结构。在美国 Thermo Scientific 公司的 Escalab 250 型 X 射线光 电子能谱仪下进行 XPS 测试, 测试结果通过 XPS PEAKER 4.1 软件进行分析。在天津先权仪器有限 公司 TP-5080 型动态吸附仪上进行 $\mathrm{H}_{2}-\mathrm{TPR}$ 测试, 测试时取 $50 \mathrm{mg}$ 样品用纯 $\mathrm{N}_{2}$ 在 $200^{\circ} \mathrm{C}$ 吹扫 $1 \mathrm{~h}$, 然 后冷却至 $50^{\circ} \mathrm{C}$, 再通入流速为 $30 \mathrm{~mL} / \mathrm{min}$ 的 $6 \%$ $\mathrm{H}_{2} / \mathrm{N}_{2}$, 以 $10^{\circ} \mathrm{C} / \mathrm{min}$ 的升温速率升温至 $800^{\circ} \mathrm{C}$ 进行 程序升温还原。

\section{2 结果与讨论}

\section{1 催化剂的 SCR 活性评价结果}

图 3 显示了不同负载量的 $\mathrm{MnO}_{2} / \mathrm{MWCNTs}$ 和 $\mathrm{MnO}_{x} / \mathrm{MWCNTs}$ 催化剂在 $80 \sim 180^{\circ} \mathrm{C}$ 范围内的脱硝 活性, 由图可知, $\mathrm{MnO}_{x} / \mathrm{MWCNTs}$ 催化剂在整个温 度区间的脱硝率都很低，在 $180^{\circ} \mathrm{C}$ 时才达到约 $70 \%$ 的脱硝率。而 $\mathrm{MnO}_{2} / \mathrm{MWCNTs}$ 催化剂具有较好的催 化活性， $120^{\circ} \mathrm{C}$ 时，催化剂的脱硝率为 $75 \%$ 92\%; $160^{\circ} \mathrm{C}$ 时，催化剂的脱硝率达到 $93 \% \sim 100 \%$, 且其催 化活性随着 $\mathrm{Mn} / \mathrm{C}$ 摩尔比的增加呈现先增加后下降 的趋势, 并在 $\mathrm{Mn} / \mathrm{C}$ 摩尔比为 $10 \%$ 时达到最大, 该

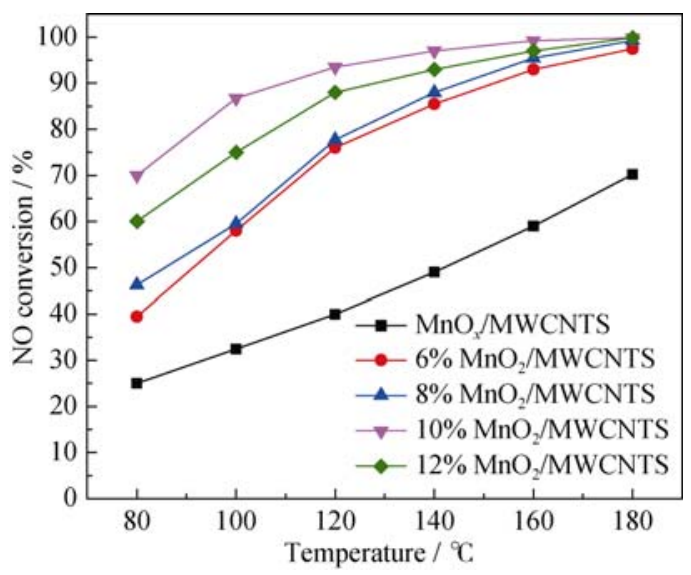

图 $3 \mathrm{MnO}_{2} / \mathrm{MWCNTs}$ 及 $\mathrm{MnO}_{x} / \mathrm{MWCNTs}$ 催化剂的 SCR 活性 Fig. 3 SCR activity of $\mathrm{MnO}_{2} / \mathrm{MWCNTs}$ catalysts and $\mathrm{MnO}_{x} /$ MWCNTs catalyst

Reaction conditions: $[\mathrm{NO}]=\left[\mathrm{NH}_{3}\right]=400 \times 10^{-6},\left[\mathrm{O}_{2}\right]=5 \%, \mathrm{~N}_{2}$ as balance gas, $\mathrm{WHSV}=210 \mathrm{~L} /\left(\mathrm{g}_{\text {cat }} \cdot \mathrm{h}\right), 200 \mathrm{mg}$ sample 
催化剂在 $80^{\circ} \mathrm{C}$ 活性高达 $70 \%, 140^{\circ} \mathrm{C}$ 即可达到 $97 \%$ 以 上的转化率。但是当进一步增大 $\mathrm{Mn} / \mathrm{C}$ 摩尔比, 催 化剂活性不再升高反而下降, 这可能是由于过高的 负载量导致活性组分发生聚集而使暴露的活性位点 减少所致。

\subsection{BET 分析}

表 1 为负载锰氧化物前后 MWCNTs 的比表面 积、孔容和平均孔径的孔结构参数。与酸化 MWCNTs 相比, $\mathrm{MnO}_{x} / \mathrm{MWCNTs}$ 催化剂的比表面积 和孔容都有所减小, 而平均孔径变大, 这可能是由 于负载的 $\mathrm{MnO}_{x}$ 颗粒填充或堵塞了载体的部分孔 道。另外, 与原始 MWCNTs 和酸化 MWCNTs 相比, 可以看出不同负载量的 $\mathrm{MnO}_{2} / \mathrm{MWCNTs}$ 催化剂的 比表面积都增大，这可以归因于形成的纳米 $\mathrm{MnO}_{2}$ 均匀地包覆在 MWCNTs 表面。值得注意的是, $10 \%$ $\mathrm{MnO}_{2} / \mathrm{MWCNTs}$ 催化剂虽然表现出最佳的低温 SCR 活性, 但它的比表面积并不是催化剂中最大的, 说 明比表面积不是影响催化剂活性的决定性因素 ${ }^{[17]}$ 。

\section{$2.3 \mathrm{X}$ 射线衍射分析}

图 4 为 $\mathrm{MnO}_{2} / \mathrm{MWCNTs} 、 \mathrm{MnO}_{x} / \mathrm{MWCNTs}$ 及原 始 MWCNTs 的 XRD 图谱, 从图中可以看出原始 MWCNTs 只在 $2 \theta=26.1^{\circ} 、 42.8^{\circ} 、 54.2^{\circ}$ 和 $77.6^{\circ}$ 附近 有明显的石墨碳衍射峰 ${ }^{[18]}$, 且这些衍射峰强度均随 着催化剂负载量的增加而减弱, 说明 $\mathrm{MnO}_{2}$ 催化剂均 匀地分散在 MWCNTs 表面, 它与碳纳米管间的相互 作用逐渐增强。此外, 不同负载量的 $\mathrm{MnO}_{2} / \mathrm{MWCNTs}$ 催化剂在 $2 \theta=12.4^{\circ} 、 36.6^{\circ}$ 和 $66.3^{\circ}$ 处有三处明显的宽衍 射峰, 归属于层状结构 $\delta-\mathrm{MnO}_{2}(\mathrm{PDF} \# 52-0556)^{[19]}$, 其 为含钾的水合二氧化锰。 $\mathrm{MnO}_{2} / \mathrm{MWCNTs}$ 催化剂都具 有较宽的衍射峰形, 这是因为反应温度为 $70^{\circ} \mathrm{C}$, 反 应速度较快, 晶体的形成和生长较快, 产物的结晶 性较差。另外, $\mathrm{MnO}_{x} / \mathrm{MWCNTs}$ 催化剂的 XRD 图谱 中显示 $\mathrm{MnO}_{x}$ 的 6 处特征衍射峰, 分别位于 $2 \theta=$ $18.1^{\circ} 、 38.9^{\circ} 、 36.1^{\circ} 、 58.6^{\circ} 、 59.8^{\circ}$ 和 $74.1^{\circ}$, 归属于 黑锰矿晶体 $\mathrm{Mn}_{3} \mathrm{O}_{4}{ }^{[20]}$ (PDF \#24-0734)。一般而言, 高氧化态的氧化锰更有利于 $\mathrm{NH}_{3}-\mathrm{SCR}$ 反应 ${ }^{[21]}$, 因 此 $\mathrm{MnO}_{2} / \mathrm{MWCNTs}$ 的低温 SCR 活性高于 $\mathrm{MnO}_{x}$ / MWCNTs 催化剂。

\subsection{FESEM 表征}

图 5 为原始 MWCNTs、 $\mathrm{MnO}_{x} / \mathrm{MWCNTs}$ 及 $10 \%$ $\mathrm{MnO}_{2} / \mathrm{MWCNTs}$ 催化剂的 FESEM 照片。比较图 5(a)、5(b)可以看出, 原始 MWCNTs 的表面较为光 滑，没有颗粒状物质; 而 $\mathrm{MnO}_{x} / \mathrm{MWCNTs}$ 催化剂部 分区域出现明显的 $\mathrm{MnO}_{x}$ 颗粒团聚现象。图 5(c)和(d) 为 $10 \% \mathrm{MnO}_{2} / \mathrm{MWCNTs}$ 催化剂在不同放大倍数下 的 FESEM 照片。在低倍 FESEM 照片上可以明显看 到原始 MWCNTs 上生长的 $\mathrm{MnO}_{2}$ 呈现层状结构, 该 形貌也符合 XRD 分析的 $\delta-\mathrm{MnO}_{2}$ 层状晶相。而从高 倍 FESEM 照片可以更加清晰地看到, 无序纳米片 为主要产物, 纳米片弯曲并且相互连接形成网络状

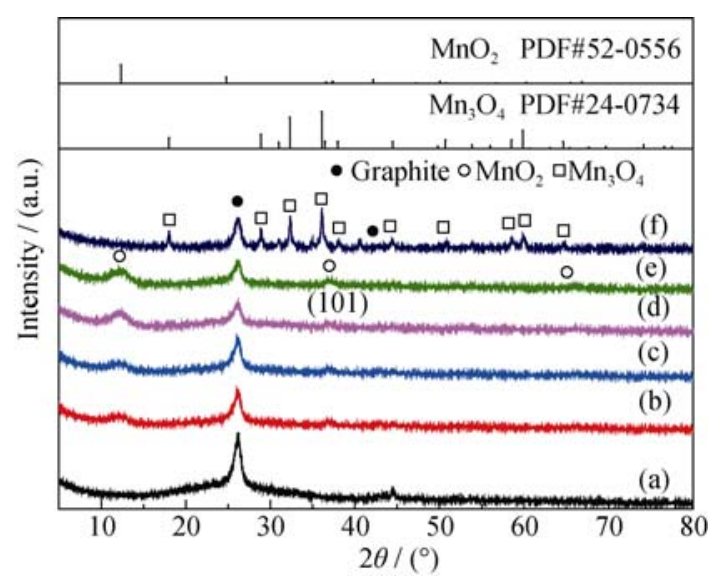

图 $4 \mathrm{MnO}_{2} / \mathrm{MWCNTs}$ 及 $\mathrm{MnO}_{x} / \mathrm{MWCNTs}$ 催化剂的 XRD 图谱 Fig. 4 XRD patterns of $\mathrm{MnO}_{2} / \mathrm{MWCNTs}$ catalysts and $\mathrm{MnO}_{x} /$ MWCNTs catalyst

(a) Pristine MWCNTs; (b) $6 \% \mathrm{MnO}_{2} / \mathrm{MWCNTs}$; (c) $8 \% \mathrm{MnO}_{2} / \mathrm{MWCNTs}$; (d) $10 \% \mathrm{MnO}_{2} / \mathrm{MWCNTs}$; (e) $12 \% \mathrm{MnO}_{2} / \mathrm{MWCNTs}$; (f) $\mathrm{MnO}_{x} / \mathrm{MWCNTs}$

表 1 原始 MWCNTs、酸化 MWCNTs 及不同催化剂样品的比表面积、孔体积、平均孔径

Table 1 BET surface area, pore volumes and average pore size of pristine MWCNTs, acid-treated MWCNTs and different catalyst samples

\begin{tabular}{cccc}
\hline Sample & $S_{\mathrm{BET}} /\left(\mathrm{m}^{2} \cdot \mathrm{g}^{-1}\right)$ & Pore volume $/\left(\mathrm{cm}^{3} \cdot \mathrm{g}^{-1}\right)$ & Average pore size $/ \mathrm{nm}$ \\
\hline Pristine MWCNTs & 72 & 0.15 & 8.1 \\
Acid-treated MWCNTs & 91 & 0.18 & 7.7 \\
$\mathrm{MnO}_{x} / \mathrm{MWCNTs}$ & 69 & 0.15 & 9.0 \\
$6 \% \mathrm{MnO}_{2} / \mathrm{MWCNTs}$ & 99 & 0.28 & 11.2 \\
$8 \% \mathrm{MnO}_{2} / \mathrm{MWCNTs}$ & 100 & 0.29 & 11.6 \\
$10 \% \mathrm{MnO}_{2} / \mathrm{MWCNTs}$ & 94 & 0.29 & 12.5 \\
$12 \% \mathrm{MnO}_{2} / \mathrm{MWCNTs}$ & 111 & 0.31 & 11.3 \\
\hline
\end{tabular}



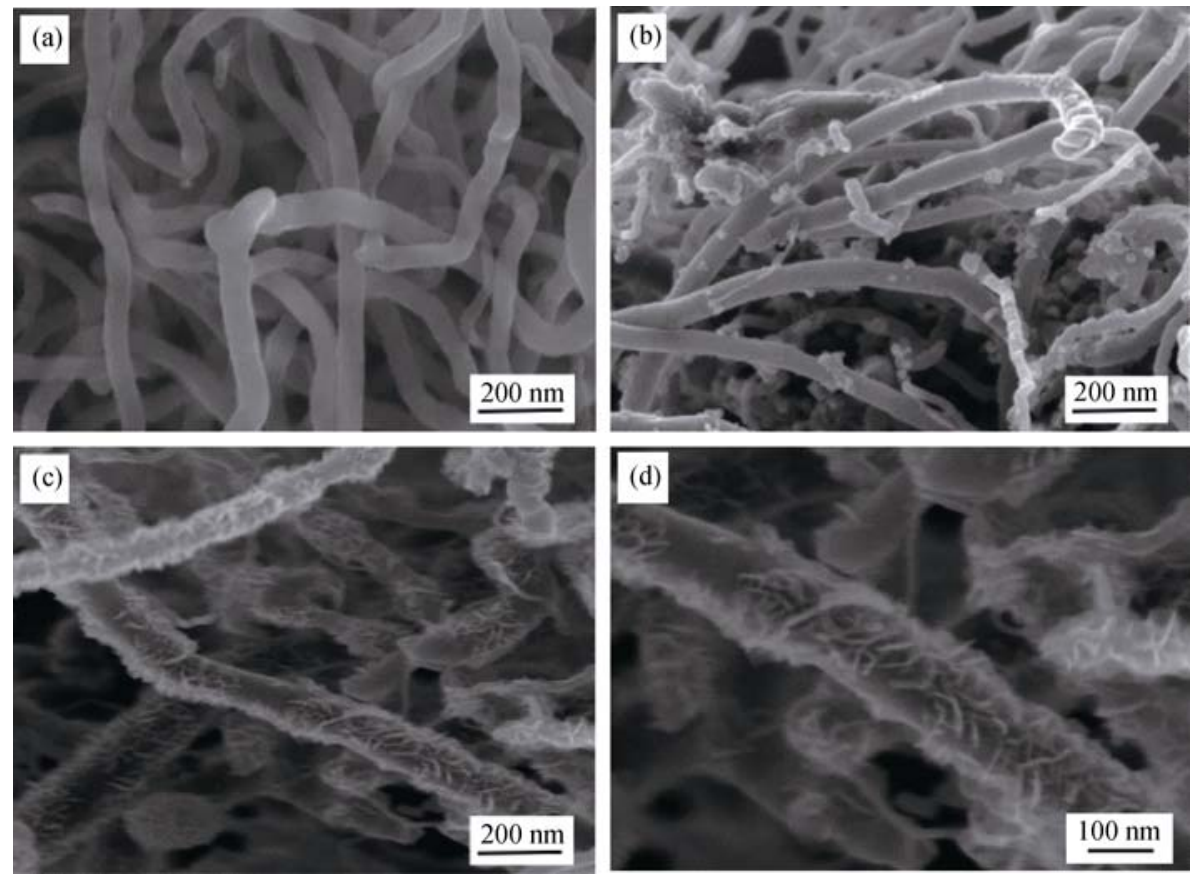

图 5 原始 MWCNTs (a), $\mathrm{MnO}_{x} / \mathrm{MWCNTs}(\mathrm{b})$ 及 $10 \% \mathrm{MnO}_{2} / \mathrm{MWCNTs}(\mathrm{c}, \mathrm{d}$ ) 催化剂的 FESEM 照片

Fig. 5 FESEM images of (a) pristine MWCNTs, (b) $\mathrm{MnO}_{x} / \mathrm{MWCNTs}$ catalyst and (c, d) $10 \% \mathrm{MnO}_{2} / \mathrm{MWCNTs}$ catalyst

的片状结构, 均匀缠绕在 MWCNTs 表面, 这种纳米 片状的 $\mathrm{MnO}_{2}$, 在 MWCNTs 表面分散得很好, 可为 催化反应提供更多的活性位点, 进而增强 $\mathrm{MnO}_{2} /$ MWCNTs 催化剂的 SCR 活性。

\section{5 透射电镜分析}

利用透射电镜对原始 MWCNTs、 $\mathrm{MnO}_{x} / \mathrm{MWCNTS}$ 及 $10 \% \mathrm{MnO}_{2}$ /MWCNTs 催化剂的微观形貌进一步 分析, 原始 MWCNTs(图 6(a))轮廓清晰, 表面无杂 质; 而 $\mathrm{MnO}_{x} / \mathrm{MWCNTs}$ 催化剂(图 6(b)) 部分区域出 现明显的 $\mathrm{MnO}_{x}$ 颗粒团聚现象, 这可能是其低温 $\mathrm{SCR}$ 活性较低的原因。另外, 从图 6(c) 可以看出, $10 \% \mathrm{MnO}_{2} / \mathrm{MWCNTs}$ 催化剂中 $\delta-\mathrm{MnO}_{2}$ 纳米薄片均 匀缠绕在 MWCNTs 表面, 这一微观形貌与 XRD 和 SEM 照片显示的结果一致。从对应的高分辨透射 分析(图 6(d)) 可以看出, $\delta-\mathrm{MnO}_{2}$ 上存在弱的晶格条 纹, 对应晶面(101), 说明 $\delta-\mathrm{MnO}_{2}$ 趋于无定型, 这 一结果与 XRD 分析相一致。为了研究 $10 \% \mathrm{MnO}_{2} /$ MWCNTs 催化剂的表面元素成分, 对图 6(c)中所 选的红色圆形区域进行能谱分析, 结果如图 6(e)所 示。从 EDX 分析可以看出, $10 \% \mathrm{MnO}_{2} / \mathrm{MWCNTS}$ 样品表面含有 $\mathrm{Mn}$ 和 $\mathrm{O}$ 两种元素 $(\mathrm{Cu}$ 元素来自于铜 网, Si 元素来自于 $\mathrm{Si}$ 漂移探测器, 表明镇氧化物 已成功负载在碳纳米管表面。并且, EDX 结果中还 存在微量 $\mathrm{K}$ 元素, 这是由于 $\delta-\mathrm{MnO}_{2}$ 生成过程中引 入到 $\mathrm{MnO}_{2}$ 的层间导致的, 而一定量的 $\mathrm{K}$ 离子有利 于稳定 $\delta-\mathrm{MnO}_{2}{ }^{[22]}$ 。

\section{$2.6 \mathrm{X}$ 射线光电子能谱分析}

为了分析催化剂表面吸附氧含量和负载镇氧化物 的价态, 对 $10 \% \mathrm{MnO}_{2} / \mathrm{MWCNTs}$ 和 $\mathrm{MnO}_{x} / \mathrm{MWCNTs}$ 催化剂进行 XPS 表征, 结果如图 7 所示。从图 7(a) 中可以看出 $10 \% \mathrm{MnO}_{2} / \mathrm{MWCNTs}$ 催化剂的 $\mathrm{Mn} 2 \mathrm{p}$ 峰分 别位于 642.3 和 $654.0 \mathrm{eV}$ 附近, 其能隙差为 $11.7 \mathrm{eV}$, 即 $\mathrm{Mn}$ 以 $\mathrm{MnO}_{2}$ 形式存在 ${ }^{[23]}$ 。而 $\mathrm{Mn}^{4+}$ 是最活跃的 $\mathrm{Mn}$ 物种, 它能促进 SCR 反应进行, 这也是催化剂 表现出优异低温 SCR 活性的主要原因。图 7(b)中 O1s 谱峰结合能位于 531.4 534.0 eV 和 529.8 530.1 eV 的 峰分别归属于晶格氧 $\mathrm{O}^{2-}$ 的峰(用 $\mathrm{O}_{\mathrm{s}}$ 表示)及表面吸 附氧峰(用 $\mathrm{O}_{\mathrm{L}}$ 表示) ${ }^{[24]}$ 。研究表明, 表面吸附氧的存在 有利于 $\mathrm{NO}$ 转化为 $\mathrm{NO}_{2}$, 进而加速 $\mathrm{SCR}$ 反应 ${ }^{[2]}$ 。

利用峰面积求得 $10 \% \mathrm{MnO}_{2} / \mathrm{MWCNTs}$ 和 $\mathrm{MnO}_{x} /$ MWCNTs 催化剂表面吸附氧与晶格氧的相对含量, 结果如表 2 所示。从表 2 可知, $10 \% \mathrm{MnO}_{2} / \mathrm{MWCNTs}$ 催 化剂的表面吸附氧含量为 $66.7 \%$, 高于 $\mathrm{MnO}_{x} / \mathrm{MWCNTS}$ 催化剂的 $61.1 \%$ 。因此可以推断出, 较高的表面吸 附氧含量是 $10 \% \mathrm{MnO}_{2} / \mathrm{MWCNTs}$ 催化剂具备较优 低温 SCR 活性的原因之一。

\section{$2.7 \mathrm{H}_{2}$-TPR 分析}

图 8 为 $\mathrm{MnO}_{x} / \mathrm{MWCNTs}$ 和 $10 \% \mathrm{MnO}_{2} / \mathrm{MWCNTs}$ 催化剂的 $\mathrm{H}_{2}$-TPR 图, 由图可知, $\mathrm{MnO}_{x} / \mathrm{MWCNTs}$ 催 化剂只在 $350 \sim 562^{\circ} \mathrm{C}$ 范围内出现一个还原峰, 此还 原峰归属于 $\mathrm{Mn}_{3} \mathrm{O}_{4}$ 向 $\mathrm{MnO}$ 的还原, 说明样品中只存 在 $\mathrm{Mn}_{3} \mathrm{O}_{4}$, 这与图 4 的结果一致。而 $\mathrm{MnO}_{2} / \mathrm{MWCNTs}$ 

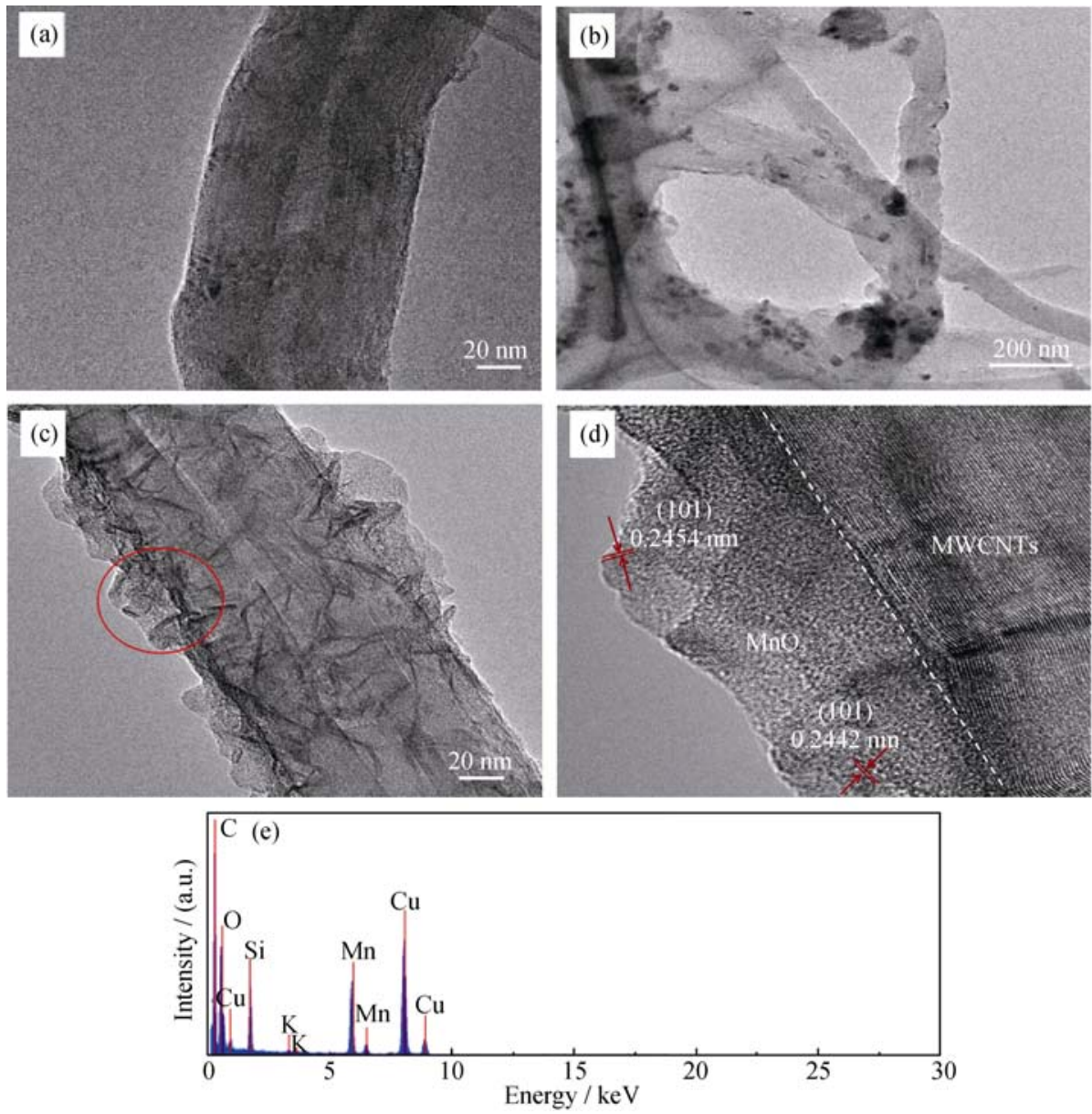

图 6 原始 MWCNTs (a), $\mathrm{MnO}_{x} / \mathrm{MWCNTs}$ (b)及 $10 \% \mathrm{MnO}_{2} / \mathrm{MWCNTs}\left(\mathrm{c}\right.$ )催化剂的 TEM 谱图和 $10 \% \mathrm{MnO}_{2} / \mathrm{MWCNTs}^{-}$ (d)催化剂的 HRTEM 图像; (e) c 中红色圆形区域的 EDX 谱图

Fig. 6 TEM images of (a) pristine MWCNTs, (b) $\mathrm{MnO}_{x} / \mathrm{MWCNTs}$ catalyst and (c) $10 \% \mathrm{MnO}_{2} / \mathrm{MWCNTs}$ catalyst and HRTEM image of $10 \% \mathrm{MnO}_{2} / \mathrm{MWCNT}$ catalyst, as well as (e) EDX pattern of $10 \% \mathrm{MnO}_{2} / \mathrm{MWCNT}$ catalyst from the annular region in (c)
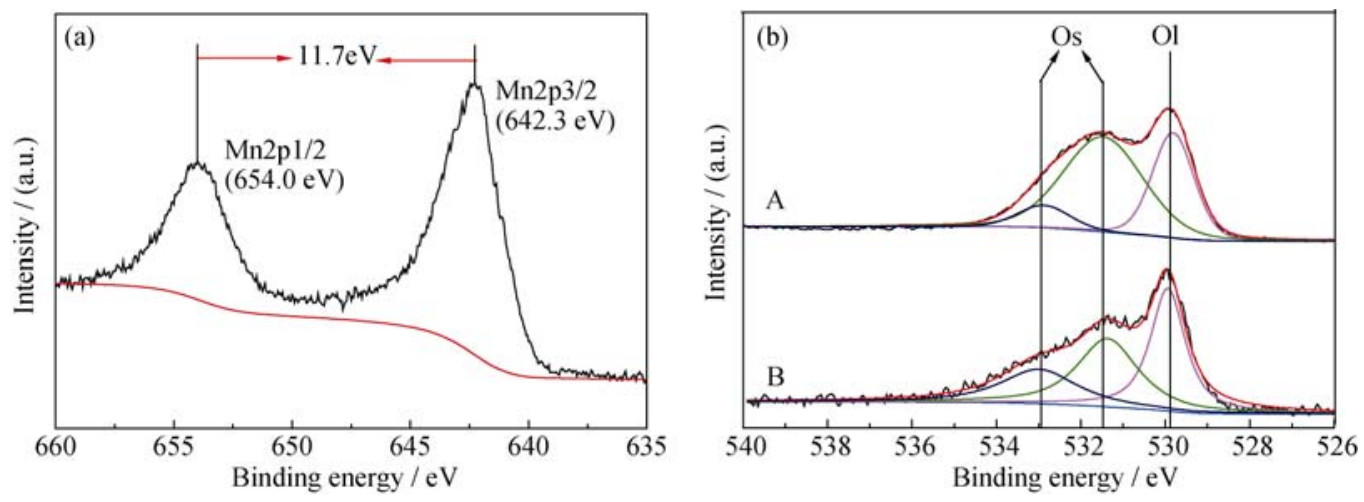

图 $710 \% \mathrm{MnO}_{2} / \mathrm{MWCNTs}$ 催化剂的 Mn2p XPS 谱图及不同催化剂样品的 O1s XPS 谱图

Fig. $7 \mathrm{Mn} 2 \mathrm{p}$ XPS spectra of $10 \% \mathrm{MnO}_{2} / \mathrm{MWCNTs}$ catalyst (a) and $\mathrm{O} 1 \mathrm{~s}$ (b) spectra of different catalyst samples (a) $\mathrm{Mn} 2 \mathrm{p}$; (b) $\mathrm{O} 1 \mathrm{~s}$, (A) $10 \% \mathrm{MnO}_{2} / \mathrm{MWCNTs}$, (B) $\mathrm{MnO}_{x} / \mathrm{MWCNTs}$

催化剂在 $181 \sim 351^{\circ} \mathrm{C}$ 和 $358 \sim 512^{\circ} \mathrm{C}$ 范围内出现了两 个还原峰, 分别归属于 $\mathrm{MnO}_{2}$ 向 $\mathrm{Mn}_{3} \mathrm{O}_{4}$ 以及 $\mathrm{Mn}_{3} \mathrm{O}_{4}$ 向 $\mathrm{MnO}$ 的还原 ${ }^{[26]}$ 。可见, 与 $\mathrm{MnO}_{x} / \mathrm{MWCNTs}$ 催化 剂相比, $10 \% \mathrm{MnO}_{2} / \mathrm{MWCNTs}$ 催化剂具有较低的还 原温度, 表明催化剂具有较好的氧化还原能力。

\section{$2.8 \mathrm{H}_{2} \mathrm{O}$ 和 $\mathrm{SO}_{2}$ 对催化剂活性的影响}

催化剂先在 $180^{\circ} \mathrm{C}$ 连续稳定反应 $1 \mathrm{~h}$ 后, 在 $180^{\circ} \mathrm{C}$ 分别考察了添加 $5 \% \mathrm{H}_{2} \mathrm{O}$ 和 $100 \times 10^{-6} \mathrm{SO}_{2}$ 的添加对 催化剂活性的影响, 结果如图 9 所示。如图 9 所示, 加入 $5 \% \mathrm{H}_{2} \mathrm{O}$ 后的 $1 \sim 6 \mathrm{~h}$ 时间内, $\mathrm{MnO}_{x} / \mathrm{MWCNTs}$ 
表 $210 \% \mathrm{MnO}_{2} / \mathrm{MWCNTs}$ 及 $\mathrm{MnO}_{x} / \mathrm{MWCNTs}$ 催化剂表面氧的相对含量

Table 2 Relative content of $\mathrm{O}$ for $10 \% \mathrm{MnO}_{2} / \mathrm{MWCNTs}$ and $\mathrm{MnO}_{x} / \mathrm{MWCNTs}$ catalyst

\begin{tabular}{ccl}
\hline Sample & $\mathrm{O}_{\mathrm{S}} / \%$ & $\mathrm{O}_{\mathrm{L}} / \%$ \\
\hline $10 \% \mathrm{MnO}_{2} / \mathrm{MWCNTs}$ & 66.7 & 33.3 \\
$\mathrm{MnO}_{x} / \mathrm{MWCNTs}$ & 61.1 & 38.9 \\
\hline
\end{tabular}

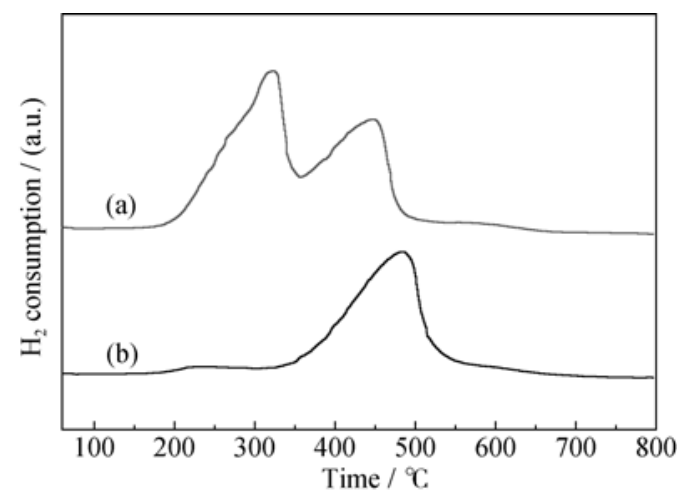

图 $810 \% \mathrm{MnO}_{2} / \mathrm{MWCNTs}(\mathrm{a})$ 及 $\mathrm{MnO}_{x} / \mathrm{MWCNTs}(\mathrm{b})$ 催化剂的 $\mathrm{H}_{2}$-TPR 图

Fig. $8 \quad \mathrm{H}_{2}$ temperature-programmed reduction $\left(\mathrm{H}_{2}-\mathrm{TPR}\right)$ profiles for $10 \% \mathrm{MnO}_{2} / \mathrm{MWCNTs}$ (a) and $\mathrm{MnO}_{x} / \mathrm{MWCNTs}$ (b) catalyst

催化剂的脱硝率由初始的 70.1\%下降至 37.6\%左右; 而 $10 \% \mathrm{MnO}_{2} / \mathrm{MWCNTs}$ 催化剂的仍能达到 $90.2 \%$, 仅下降了 $10 \%$ 左右。切断 $\mathrm{H}_{2} \mathrm{O}$ 后, $10 \% \mathrm{MnO}_{2} / \mathrm{MWCNTs}$ 催化剂的脱硝效率恢复至初始水平 $100 \%, \mathrm{MnO}_{x}$ / MWCNTs 催化剂的脱硝效率恢复至 $54.5 \%$ 。以上结 果表明, $10 \% \mathrm{MnO}_{2} / \mathrm{MWCNTs}$ 催化剂具有较好的抗 $\mathrm{H}_{2} \mathrm{O}$ 性能。

由图 9 可知, 经过 $6 \mathrm{~h}$ 的抗硫实验两种催化剂的 脱硝率均有下降, 且 $10 \% \mathrm{MnO}_{2} / \mathrm{MWCNTs}$ 催化剂活 性下降幅度明显小于 $\mathrm{MnO}_{x} / \mathrm{MWCNTs}$ 催化剂。切断

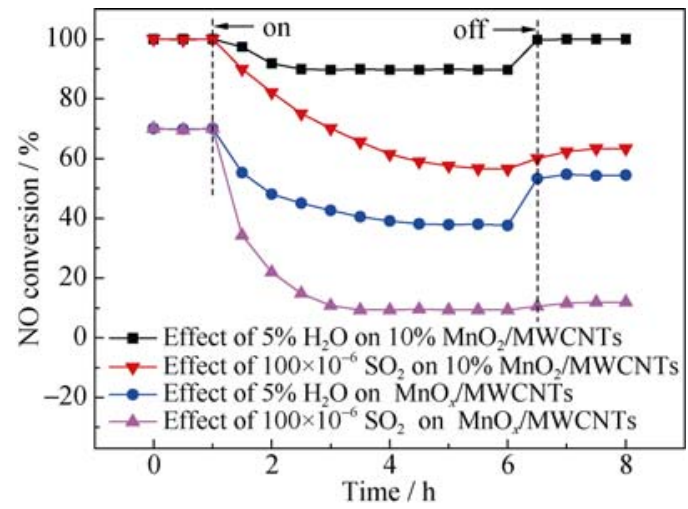

图 $9 \mathrm{H}_{2} \mathrm{O}$ 和 $\mathrm{SO}_{2}$ 对催化剂 $\mathrm{SCR}$ 活性的影响

Fig. 9 Effect of $\mathrm{H}_{2} \mathrm{O}$ and $\mathrm{SO}_{2}$ on the SCR activities of catalysts at $180^{\circ} \mathrm{C}$

Reaction conditions: $[\mathrm{NO}]=\left[\mathrm{NH}_{3}\right]=400 \times 10^{-6},\left[\mathrm{O}_{2}\right]=5 \%,\left[\mathrm{H}_{2} \mathrm{O}\right]=5 \%$, $\left[\mathrm{SO}_{2}\right]=100 \times 10^{-6}, \mathrm{~N}_{2}$ as balance gas, WHSV $=210 \mathrm{~L} /\left(\mathrm{g}_{\text {cat }} \cdot \mathrm{h}\right), 200 \mathrm{mg}$ sample
$\mathrm{SO}_{2}$ 后, $10 \% \mathrm{MnO}_{2} / \mathrm{MWCNTs}$ 和 $\mathrm{MnO}_{x} / \mathrm{MWCNTs}$ 催 化剂的脱硝率分别恢复 $7 \%$ 和 $3 \%$ 。可见，相对于 $\mathrm{MnO}_{x} / \mathrm{MWCNTs}$ 催化剂, $10 \% \mathrm{MnO}_{2} / \mathrm{MWCNTs}$ 催化 剂具有较好的抗 $\mathrm{SO}_{2}$ 性能。

\section{3 结论}

采用一种新颖的氧化还原沉淀法制备了 $\mathrm{MnO}_{2} /$ MWCNTs 脱硝催化剂，该方法制备过程简单，耗时 短, 且环境友好。在 $\mathrm{MnO}_{2} / \mathrm{MWCNTs}$ 催化剂中, 其 负载的纳米片状 $\mathrm{MnO}_{2}$ 能够均匀缠绕在 MWCNTs 表面，催化活性测试结果表明, $\mathrm{MnO}_{2} / \mathrm{MWCNTs}$ 催 化剂具有良好的低温 $\mathrm{SCR}$ 活性，温度为 $160^{\circ} \mathrm{C}$ 时， $\mathrm{NO}_{x}$ 最高转化率均高于 $93 \%$ 。其中，当 $\mathrm{Mn} / \mathrm{C}$ 摩尔 比为 $10 \%$ 时, $\mathrm{MnO}_{2} / \mathrm{MWCNTs}$ 催化剂呈现最好的低 温 SCR 活性, $140^{\circ} \mathrm{C}$ 可达到 $97 \%$ 以上的转化率。另外, 与传统等体积浸渍法制备的 $\mathrm{MnO}_{x} / \mathrm{MWCNTs}$ 催化 剂相比, $10 \% \mathrm{MnO}_{2} / \mathrm{MWCNTs}$ 催化剂中具有较好的 抗 $\mathrm{H}_{2} \mathrm{O}$ 和抗 $\mathrm{SO}_{2}$ 性能。

\section{参考文献:}

[1] WU D W, ZHANG Q L, LIN T, et al. Effect of Fe on the selective catalytic reduction of $\mathrm{NO}$ by $\mathrm{NH}_{3}$ at low temperature over $\mathrm{Mn} / \mathrm{CeO}_{2}-\mathrm{TiO}_{2}$ catalyst. J. Inorg. Mater, 2012, 27(5): 495-500.

[2] GAO R H, ZHANG D S, LIU X G, et al. Enhanced catalytic performance of $\mathrm{V}_{2} \mathrm{O}_{5}-\mathrm{WO}_{3} / \mathrm{Fe}_{2} \mathrm{O}_{3} / \mathrm{TiO}_{2}$ microspheres for selective catalytic reduction of $\mathrm{NO}$ by $\mathrm{NH}_{3}$. Catal. Sci. Technol., 2013, 3(1): 191-199.

[3] NIE J Y, WU X D, MA Z R, et al. Tailored temperature window of $\mathrm{MnO}_{x}-\mathrm{CeO}_{2} \mathrm{SCR}$ catalyst by addition acidic metal oxides. Chin. J. Catal., 2014, 35(8): 1281-1288.

[4] FANG C, ZHANG D S, CAI S X, et al. Low-temperature selective catalytic reduction of $\mathrm{NO}$ with $\mathrm{NH}_{3}$ over nanoflaky $\mathrm{MnO}_{x}$ on carbon nanotubes in situ prepared via a chemical bath deposition route. Nanoscale, 2013, 5(19): 9199-9207.

[5] POURKHALIL M, MOGHADDAM A Z, RASHIDI A, et al. Preparation of highly active manganese oxides supported on functionalized MWNTs for low temperature $\mathrm{NO}_{x}$ reduction with $\mathrm{NH}_{3}$. Appl. Surf. Sci., 2013, 279: 250-259.

[6] YAO G H, WANG F, WANG X B, et al. Magnetic field effects on selective catalytic reduction of $\mathrm{NO}$ by $\mathrm{NH}_{3}$ over $\mathrm{Fe}_{2} \mathrm{O}_{3}$ catalyst in a magnetically fluidized bed. Energy, 2010, 35(5): 2295-2300.

[7] KAPTEIJN F, SINGOREDJO L, ANDREINI A. Activity and selectivity of pure manganese oxides in the selective catalytic reduction of nitric oxide with ammonia. Appl. Catal. B: Environ., 1994, 3(2): 173-189. 
[8] QI G S, YANG R T, CHANG R. $\mathrm{MnO}_{x}-\mathrm{CeO}_{2}$ mixed oxides prepared by co-precipitation for selective catalytic reduction of $\mathrm{NO}$ with $\mathrm{NH}_{3}$ at low temperatures. Appl. Catal. B: Environ., 2004, 51(2): 93-106.

[9] LI J H, CHEN J J, KE R, et al. Effects of precursors on the surface $\mathrm{Mn}$ species and the activities for $\mathrm{NO}$ reduction over $\mathrm{MnO}_{x} / \mathrm{TiO}_{2}$ catalysts. Catal. Commun., 2007, 8(12): 1896-1900.

[10] KIJLSTRA W S, BRANDS D S, POELS E K, et al. Mechanism of the selective catalytic reduction of $\mathrm{NO}$ by $\mathrm{NH}_{3}$ over $\mathrm{MnO}_{x} / \mathrm{Al}_{2} \mathrm{O}_{3}$. Catal., 1997, 171(1): 208-218.

[11] SHEN B X, GUO B B, WU C F, et al. Low temperature selective catalytic reduction of $\mathrm{NO}$ by $\mathrm{MnO}_{x} / \mathrm{ACF}$. Environ. Pollut. Control, 2006, 28(11): 801-803.

[12] WANG $\mathrm{H} \mathrm{J}$, WANG X $\mathrm{H}$, ZHENG $\mathrm{J} \mathrm{D}$, et al. $\mathrm{Pt} / \mathrm{MoO}_{3}-$ $\mathrm{WO}_{3} / \mathrm{CNTs}$ catalyst with excellent performance for methanol electrooxidation. Chinese J. Catal., 2014, 35(10): 1687-1694.

[13] TAN Z Q, ABE H, NAITO M, et al. Arrangement of palladium nanoparticles templated by supramolecular self-assembly of SDS wrapped on single-walled carbon nanotubes. Chem. Commun., 2010, 46(24): 4363-4365.

[14] MA S B, AHN KY, LEE E S, et al. Synthesis and characterization of manganese dioxide spontaneously coated on carbon nanotubes. Carbon, 2007, 45(2): 375-382.

[15] JIN X B, ZHOU W Z, ZHANG S W, et al. Nanoscale microelectro chemical cells on carbon nanotubes. Small, 2007, 3(9): 1513-1517.

[16] WANG L S, HUANG B C, SU Y X, et al. Manganese oxides supported on multi-walled carbon nanotubes for selective catalytic reduction of $\mathrm{NO}$ with $\mathrm{NH}_{3}$ : catalytic activity and characterization. Chem. Eng. J., 2012, 192: 232-241.

[17] WANG X, ZHENG Y Y, XU Z, et al. Amorphous $\mathrm{MnO}_{2}$ supported on carbon nanotubes as a superior catalyst for low temperature NO reduction with $\mathrm{NH}_{3}$. Rsc. Adv., 2013, 3(29): 11539-11542.
[18] HU J, YUAN A B, WANG Y Q, et al. Improved cyclability of nano- $\mathrm{MnO}_{2} / \mathrm{CNT}$ composite supercapacitor electrode derived from room-temperature solid reaction. Acta Phys. -Chim. Sin., 2009, 25(5): 987-993.

[19] ZHU G, DENG L J, WANG J F, et al. Hydrothermal preparation and the capacitance of hierarchical $\mathrm{MnO}_{2}$ nanoflower. Colloid. Surface. A, 2013, 434: 42-48.

[20] BAYKAL A, KAVAS H, DURMUS Z, et al. Sonochemical synthesis and chracterization of $\mathrm{Mn}_{3} \mathrm{O}_{4}$ nanoparticles. Cent. Eur. J. Chem., 2010, 8(3): 633-638.

[21] ZHANG Y B, ZHENG Y Y, WANG X, et al. Fabrication of $\mathrm{Mn}-\mathrm{CeO}_{x} / \mathrm{CNTs}$ catalysts by a redox method and their performance in low-temperature $\mathrm{NO}$ reduction with $\mathrm{NH}_{3}$. $R S C A d v$., 2015, 5(36): 28385-28388

[22] JULIEN C M, MASSOT M, POINSIGNON C. Lattice vibrations of manganese oxides: Part I. Periodic structures. Spectrochim. Acta A, 2004, 60(3): 689-700.

[23] DAI Y, LI J H, PENG Y, et al. Effects of $\mathrm{MnO}_{2}$ crystal structure and surface property on the $\mathrm{NH}_{3}-\mathrm{SCR}$ reaction at low temperature. Acta Phys. Chim. Sin., 2012, 28(7): 1771-1776.

[24] WANG Y L, WANG X J, ZHAN L, et al. Structure control of $\mathrm{V}_{2} \mathrm{O}_{5} / \mathrm{CNFs} /$ cordierite monolith catalyst and its catalytic performance on NO removal from flue gas. J. Inorg. Mater., 2012, 27(8): 800-806.

[25] LIU F D, HE H, DING Y, et al. Effect of manganese substitution on the structure and activity of iron titanate catalyst for the selective catalytic reduction of $\mathrm{NO}$ with $\mathrm{NH}_{3}$. Appl. Catal. B: Environ., 2009, 93(1): 194-204.

[26] ETTIREDDY P R, ETTIREDDY N, MAMEDOV S, et al. Surface characterization studies of $\mathrm{TiO}_{2}$ supported manganese oxide catalysts for low temperature $\mathrm{SCR}$ of $\mathrm{NO}$ with $\mathrm{NH}_{3}$. Appl. Catal. B, 2007, 76(1/2): 123-134. 